\title{
Charge of Circulating Immune Complexes as a Factor in Glomerular Basement Membrane Localization in Mice
}

\author{
Gloria R. Gallo, Teresa Caulin-Glaser, and Michael E. Lamm, \\ Department of Pathology, New York University School of Medicine, \\ New York 10016
}

\begin{abstract}
A B S T R A C T The effect of the charge of circulating immune complexes on glomerular localization was studied in a model of passive serum sickness. Preformed immune complexes of heterogeneous or restricted charge, prepared in vitro from isoelectrically focused or chemically modified proteins, were injected intravenously into mice. The distribution of immune complexes in the kidney was compared by immunofluorescence and electron microscopy. Cationic but not anionic or electrophoretically heterogeneous immune complexes gave rise to diffuse localization in the glomerular basement membrane. The binding in subepithelial and subendothelial sites correlated with the known distribution of structural anionic sites. The observations suggest that electrostatic interactions between fixed anionic sites and immune complexes may be an important factor in glomerular trapping. Alternative mechanisms based on initial localization of excess free cationic antigen cannot be completely excluded and are also considered.
\end{abstract}

\section{INTRODUCTION}

Granular deposits of immunoglobulin (Ig) demonstrable by immunofluorescence in the glomeruli of man and animals with glomerulonephritis are generally regarded as evidence of an immunologically mediated disease resulting from the deposition of immune complexes (IC). ${ }^{1}$ Deposits are believed to result from two mechanisms: glomerular trapping of circulating soluble IC (1-4), or in situ formation of immune deposits by fixation of antibody $(\mathrm{Ab})$ to structural $(5-9)$ or planted

This work was presented in part at the 13th Annual Meeting of the American Society of Nephrology, Washington, D. C., 23-25 November 1980.

Received for publication 15 May 1980 and in revised form 2 January 1981.

${ }^{1}$ Abbreviations used in this paper: $\mathrm{Ab}$, antibody; $\mathrm{Ag}$, antigen; BGG, bovine gamma globulin; IC, immune complex; PBS, 0.01 M phosphate- $0.15 \mathrm{M} \mathrm{NaCl}$, pH 7.0-7.5; RGG rabbit gamma globulin.
(10, 11) glomerular basement membrane antigens (Ag). Some diseases are characterized by deposits only in subepithelial sites, while in others, subendothelial and/or mesangial deposits predominate. The factors that determine the sites of IC localization are poorly understood. Repeated studies in experimental animals given intravenous injections of preformed soluble IC (passive serum sickness) have shown that IC lodge predominantly in the glomerular mesangium and occasionally in subendothelial sites (12-20). Localization in subepithelial sites of the glomerular basement membrane is exceptional (21). On the other hand, injection of $\mathrm{Ab}$ to renal tubular $\mathrm{Ag}$ in the model of passive Heymann nephritis has resulted in deposition of $\mathrm{Ab}$ only in subepithelial sites immediately after injection $(5,6,8)$ and in vitro perfusion of the kidney (7). These observations have suggested that subepithelial deposits, such as those found in membranous glomerulonephritis in man and animals, formerly believed to result from the deposition of circulating IC, result instead from in situ binding of $\mathrm{Ab}$ to $\mathrm{Ag}$.

Previous studies have documented the presence in the glomerular basement membrane of fixed negatively charged sites $(22,23)$ which facilitate the penetration and binding of positively charged macromolecules while retarding the penetration of negatively charged ones $(24,25)$. In the present study, the hypothesis that charge-dependent factors might influence the glomerular basement membrane localization of intravenously injected IC on a similar basis was tested in a model of passive serum sickness. The results demonstrate that cationic, but not anionic or heterogeneous IC localize in subepithelial and subendothelial sites.

\section{METHODS}

Preparation of antibody. Rabbits were hyperimmunized with bovine gamma globulin (BGG) (Pentex, Fraction II) in complete Freund's adjuvant (Difco Laboratories, Detroit, Mich.).

Isoelectric focusing. Rabbit anti-BGG antiserum was heat inactivated at $56^{\circ} \mathrm{C}$ for $30 \mathrm{~min}$ and an Ig-containing fraction 
was prepared by precipitation in $50 \%$ saturated $\left(\mathrm{NH}_{4}\right)_{2} \mathrm{SO}_{4}$ followed by dialysis against first $0.01 \mathrm{M}$ phosphate- $0.15 \mathrm{M}$ $\mathrm{NaCl}$ (PBS) and then 0.1\% ampholytes, pH 2-11 (Brinkmann Instruments, Inc., Westbury, N. Y.) in $\mathrm{H}_{2} \mathrm{O}$. The Ig was added to an aqueous slurry of Sephadex G-75 superfine made $2.5 \%$ ( $\mathrm{vol} / \mathrm{vol})$ in ampholytes. Preparative isoelectric focusing was carried out in a Desaga/Brinkmann double chamber for thin layer electrophoresis at $400 \mathrm{~V}$ for $20 \mathrm{~h}$, then $800 \mathrm{~V}$ for $3 \mathrm{~h} . \mathrm{pH}$ determinations were made at $1 \mathrm{~cm}$ intervals; $1 \mathrm{~cm}$ strips were mixed with $10 \mathrm{ml}$ of saline, and the protein was harvested by suctioning through scintered glass filters. Fractions were separately dialyzed against normal saline and stored at $-20^{\circ} \mathrm{C}$. The amount of $\mathrm{Ab}$ in each $\mathrm{pH}$ range was determined by quantitative precipitation with the particular BGG to be used to prepare the IC (see below).

Differently charged fractions of BGG were prepared by isoelectric focusing in the same manner and were tested for the presence of IgGl and IgG2 by double immunodiffusion with subclass-specific antisera kindly provided by Dr. John E. Butler of the University of Iowa College of Medicine.

Chemical modification of BGG antigen. Anionization (26) of BGG was done by dissolving $500 \mathrm{mg}$ in $50 \mathrm{ml} \mathrm{PBS,} \mathrm{pH} \mathrm{7.0.}$ Maleic anhydride (Fisher Scientific Co., Inc., Pittsburgh, Pa.), $1 \mathrm{gm}$, was added at $0^{\circ} \mathrm{C}$; the $\mathrm{pH}$ was maintained at 7.0 with $2 \mathrm{~N}$ $\mathrm{NaOH}$ for $10 \mathrm{~min}$. The solution was dialyzed against multiple changes of the same buffer, pH 7.5. For cationization (27) of BGG, $200 \mathrm{mg}$ was dissolved in $2.5 \mathrm{ml}$ of $0.01 \mathrm{M} \mathrm{NaCl}$ at room temperature. The solution was added to $4.4 \mathrm{ml}$ of $2 \mathrm{M}$ ethylenediamine (Fisher Scientific Co., Inc) and adjusted to pH 7.0 with $2 \mathrm{~N} \mathrm{HCl}$. While stirring, $400 \mathrm{mg}$ of 1-ethyl-3-(3-dimethylaminopropyl)carbodiimide- $\mathrm{HCl}$ (Pierce Chemical Co., Rockford, Ill.) was dissolved; the $\mathrm{pH}$ was maintained at 7.0 with $0.2 \mathrm{~N} \mathrm{HCl}$ for $2 \mathrm{~h}$. The reaction mixture was kept overnight at room temperature in a stoppered flask, and then dialyzed against several changes of normal saline.

Gel electrophoresis. The relative electrophoretic mobilities of the antigens and antibodies used to prepare IC were compared at $\mathrm{pH} 8.6$ in agarose gels (Fig. 1).

Preparation of immune complexes. The equivalence point of each fraction of rabbit $\mathrm{Ab}$ was determined by quantitative precipitation (28) for each preparation of BGG Ag. $\mathrm{E}_{280}^{1 \%}=14$ was used to calculate the amounts of $\mathrm{Ag}$ and $\mathrm{Ab}$. Since it was not possible to solubilize immune precipitates at equivalence by adding excess $\mathrm{Ag}$ and maintain solubility at neutral $\mathrm{pH}$, soluble IC were formed by slowly adding $\mathrm{Ab}$ to the calculated amount of Ag (fivefold excess) while constantly stirring at room temperature. The solutions were adjusted to $\mathrm{pH} 7.2$ with $\mathrm{HCl}$ or $\mathrm{NaOH}$, left overnight at $4^{\circ} \mathrm{C}$, and centrifuged at $12,317 \mathrm{~g}$ maximum for $10 \mathrm{~min}$ just before injection to remove small amounts of precipitate.

10 types of soluble IC were prepared from the various forms of $\mathrm{Ag}$ and $\mathrm{Ab}$ (Table $\mathrm{I}$ ): (1) native electrophoretically heterogeneous Ag (unfocused, unmodified BGG) combined with the $\left(\mathrm{NH}_{4}\right)_{2} \mathrm{SO}_{4}$ fraction of native unfocused $\mathrm{Ab},(2)$ chemically modified anionic $\mathrm{Ag}$ and focused anionic $\mathrm{Ab}$ ( $\mathrm{Ab}$ isoelectric point (pI) 2.5-5.5), (3) focused anionic $\mathrm{Ag}$ and $\mathrm{Ab}$ (both $\mathrm{Ag}$ and $\mathrm{Ab}$ pI 2.5-5.5), (4) chemically modified cationic $\mathrm{Ag}$ and focused cationic $\mathrm{Ab}(\mathrm{Ab} \mathrm{pI} 8-10),(5)$ chemically modified cationic Ag and focused neutral Ab (Ab pI 6.5-7.0), (6) chemically modified cationic $\mathrm{Ag}$ and unfocused electrophoretically heterogeneous $\mathrm{Ab}$, (7) focused cationic $\mathrm{Ag}$ and $\mathrm{Ab}$ (both $\mathrm{Ag}$ and $\mathrm{Ab}$ oI 8-10), (8) electrophoretically heterogeneous Ag and focused cationic Ab (Ab pI 8-10), (9) chemically modified anionic Ag and focused cationic $\mathrm{Ab}$ (Ab pI 8-10), (10) chemically modified cationic Ag and focused anionic Ab (Ab pI 2.5-5.5). Noncomplexed proteins given intravenously in equivalent amounts to control mice were (11) normal BGG and normal RGG or (12) chemically modified cationic Ag and

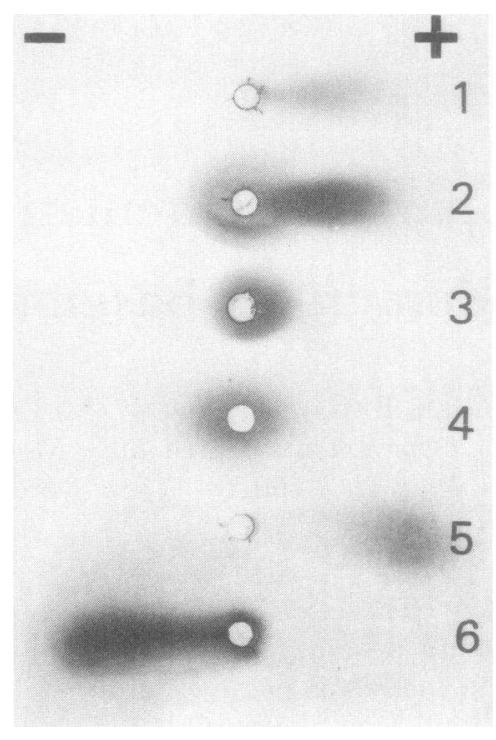

Figure 1 Agarose gel electrophoresis in $0.06 \mathrm{M}$ Na barbital, pH 8.6, after Coomassie Blue staining to show the relative mobilities of the antibodies (wells 1-3) and antigens (wells 4-6) used to prepare immune complexes. The rabbit antibodies are from $\left(\mathrm{NH}_{4}\right)_{2} \mathrm{SO}_{4}$ precipitates of antiserum against BGG. (1) Anionic fraction prepared by isoelectric focusing; (2) unfocused heterogeneous fraction; (3) cationic fraction prepared by isoelectric focusing; (4) unmodified heterogeneous BGG; (5) chemically modified anionic BGG; and (6) chemically modified cationic BGG. Focused unmodified BGG is less highly charged than chemically modified BGG by the above technique. Also, by isoelectric focusing in $6 \mathrm{M}$ urea, chemically modified anionic, and cationic BGG are about $0.4 \mathrm{pH} \mathrm{U}$ more acidic and $1.4 \mathrm{pH} \mathrm{U}$ more basic, respectively than the focused anionic and cationic BGG.

normal RGG. Isoelectric focusing could not be used to compare soluble IC directly because the chemically modified BGG were insoluble in the ampholyte solutions.

In the 10 different interacting groups used (see above), the percentage of specific $A b$ to $B G G$ in each RGG fraction ranged between 6 and $26 \%$. The $\mathrm{Ab} / \mathrm{Ag}$ ratio at equivalence was 7.1 for group 1 , which employed unaltered and unfocused $\mathrm{Ab}$ and $\mathrm{Ag}$, and was lower in the other groups that ranged between 2.1 and 5.7. These lower ratios reflect the selection brought about by isoelectric focusing and the loss of antigenic determinants by modification.

Ultracentrifugation of $I C$. Sizing of IC was performed by centrifugation through a $10-40 \%$ linear sucrose density gradient in PBS, pH 7.2, with a $60 \%$ sucrose cushion. Soluble IC in $0.2-0.3 \mathrm{ml}$ containing $0.5 \mathrm{mg}$ specific $\mathrm{Ab}$ and fivefold excess of $\mathrm{Ag}$ (total protein $15-30 \mathrm{mg}$ ) were run at $30,000 \mathrm{rpm}$ for $16 \mathrm{~h}$ in a Spinco model L5-65 ultracentrifuge in an SW 50.1 rotor. Human IgM and BGG were used as $19 \mathrm{~S}$ and $7 \mathrm{~S}$ markers, respectively. Fractions were collected dropwise from the bottom, diluted, and protein content was determined by OD $280 \mathrm{~nm}$ and double immunodiffusion. All preparations of IC yielded multiple peaks sedimenting between $9-25 \mathrm{~S}$. Selected profiles are shown in Fig. 2.

Injection protocol. Male BALB/c mice (Charles River Breeding Laboratories, Inc., Wilmington, Mass.) 6-8 wk old, weighing $25 \mathrm{~g}$ were used. In preliminary studies, a number of different injection protocols were tried with soluble IC com- 
TABLE I

Glomerular Localization of RGG and BGG 1 h after Intravenous Injection

of Immune Complexes or Noncomplexed BGG and RGG

\begin{tabular}{|c|c|c|c|c|c|c|c|}
\hline \multirow[b]{2}{*}{ Group } & \multirow[b]{2}{*}{ Substances injected ${ }^{*}$} & \multirow[b]{2}{*}{$\begin{array}{l}\text { Number } \\
\text { of mice }\end{array}$} & \multicolumn{2}{|c|}{$\begin{array}{l}\text { Immunoreactants } \\
\text { in glomeruli }\end{array}$} & \multicolumn{3}{|c|}{ Ultrastructural sites of deposits } \\
\hline & & & BGG & RGG & $\begin{array}{l}\text { Subepi- } \\
\text { thelial }\end{array}$ & $\begin{array}{l}\text { Subendo- } \\
\text { thelial }\end{array}$ & Mesangial \\
\hline \multicolumn{8}{|c|}{ Heterogeneous IC } \\
\hline 1 & Elect Het Ag/Elect Het Ab & 15 & $2+\mathrm{Mes}$ & $2+\operatorname{Mes}$ & - & - & $2+$ \\
\hline \multicolumn{8}{|l|}{ Anionic IC } \\
\hline 2 & Chem Mod An Ag/Foc An Ab & 6 & $1+$ Mes & $1+$ Mes & - & - & $1+$ \\
\hline 3 & Foc An Ag/Foc An Ab & 2 & $1+$ Mes & $1+$ Mes & - & - & $1+$ \\
\hline \multicolumn{8}{|l|}{ Cationic IC $\$$} \\
\hline 4 & Chem Mod Cat Ag/Foc Cat Ab & 9 & $3+\mathrm{GBM}$ & $3+\mathrm{GBM}$ & $3+$ & $3+$ & $2+$ \\
\hline 5 & Chem Mod Cat Ag/Foc Neut Ab & 2 & $3+\mathrm{GBM}$ & $2-3+\mathrm{GBM}$ & $1+$ & $2+$ & $2+$ \\
\hline 6 & Chem Mod Cat Ag/Elect Het Ab & 2 & $3+\mathrm{GBM}$ & $2-3+\mathrm{GBM}$ & $1+$ & $2+$ & $2+$ \\
\hline 7 & Foc Cat Ag/Foc Cat Ab & 8 & $2+$ Mes & $2+$ Mes & - & - & $1+$ \\
\hline 8 & Elect Het Ag/Foc Cat Ab & 3 & $2+$ Mes & $2+$ Mes & - & - & $1+$ \\
\hline \multicolumn{8}{|l|}{ Mixed IC } \\
\hline 9 & Chem Mod An Ag/Foc Cat Ab & 2 & $1+$ Mes & $1+$ Mes & - & - & $1+$ \\
\hline 10 & Chem Mod Cat Ag/Foc An Ab & 7 & $3+\mathrm{GBM}$ & $1+$ Mes & $1+$ & $1+$ & $2+$ \\
\hline \multicolumn{8}{|c|}{ Noncomplexed proteins } \\
\hline 11 & Nor BGG/Nor RGG & 6 & $1+$ Mes & $1+$ Mes & - & - & - \\
\hline 12 & Chem Mod Cat Ag/Nor RGG & 3 & $3+\mathrm{GBM}$ & $1+$ Mes & $1+$ & $3+$ & $2+$ \\
\hline
\end{tabular}

* Abbreviations used in this table: (1) Elect Het Ag/Elect Het Ab, electrophoretically heterogeneous antigen (unfocused, unmodified BGG) and the native unfocused electrophoretically heterogeneous antibody; (2) Chem Mod An Ag/Foc An Ab, chemically modified anionic antigen and focused anionic Ab; (3) Foc An Ag/Foc An Ab, focused anionic antigen and antibody; (4) Chem Mod Cat Ag/Foc Cat Ab, chemically modified cationic antigen and focused cationic antibody; (5) Chem Mod Cat Ag/ Foc Neut Ab, chemically modified cationic antigen and focused neutral antibody; (6) Chem Mod Cat Ag/Elect Het Ab, chemically modified cationic antigen and unfocused electrophoretically heterogeneous antibody; (7) Foc Cat Ag/Foc Cat Ab, focused cationic antigen and antibody; (8) Elect Het Ag/Foc Cat Ab, electrophoretically heterogeneous antigen and focused cationic antibody; (9) Chem Mod An Ag/Foc Cat Ab, chemically modified anionic antigen and focused cationic antibody; (10) Chem Mod Cat Ag/Foc An Ab, chemically modified cationic antigen and focused anionic antibody; (11) Nor BGG/Nor RGG, normal BGG and normal RGG; (12) Chem Mod Cat AG/Nor RGG, chemically modified cationic antigen and normal RGG.

$\$$ Determined by immunofluorescence; all animals in a given group had the same result. Mes, mesangium; GBM, glomerular basement membrane.

\$ The complexes under this subheading (groups 4-8) all have a net positive charge. Since Ag is in excess and is therefore the major determinant of net charge and because the chemically modified cationic Ag is the most cationic preparation used in this study (more cationic than the focused cationic Ag), complexes 4-6 are more cationic than complexes 7, 8. Group 4 complexes are the most cationic of all.

posed of native heterogeneous $\mathrm{Ag}$ and $\mathrm{Ab}$. A comparison by immunofluorescence was made of the amount and distribution of deposits in glomeruli. Variables included the Ag to Ab ratio (between 5- and 20-fold excess Ag), the amount of Ab per injection (between 0.25 and $6 \mathrm{mg}$ ), the number of injections (1-3) over a $24-\mathrm{h}$ period, and the time of sacrifice $(0 \mathrm{~min}$ to $6 \mathrm{~h}$ after the last injection). The maximum deposition was generally seen $1 \mathrm{~h}$ after injection. Accumulation of IC was always restricted to the mesangium and the amount was not appreciably greater after three than one injection. Also, there were no consistent differences in animals given IC prepared at different equivalence ratios. The regimen eventually chosen was one injection of IC containing $0.5 \mathrm{mg}$ of specific rabbit $\mathrm{Ab}$ and a fivefold excess of BGG Ag given over 1-2 min by tail vein in a volume of $0.3-0.5 \mathrm{ml}$. Mice were sacrificed $1 \mathrm{~h}$ later.

Tissue processing. Portions of kidney were fixed in $10 \%$ buffered formalin or Zenker's Fu 48 solution (Technicon Instruments Corp., Tarrytown, N. Y.). Paraffin embedded blocks cut at $2-3 \mu \mathrm{m}$ were stained with hematoxylin and eosin or periodic acid-Schiff for light microscopic examination. For immunofluorescence studies, multiple blocks were snap frozen in 2-methylbutane suspended in liquid nitrogen, and stored at $-70^{\circ} \mathrm{C}$. Cryostat sections $(4 \mu \mathrm{m})$ were fixed in acetone or in $10 \%$ buffered formalin for $10 \mathrm{~s}$, washed in PBS, incubated in a moist chamber with antiserum, and finally washed in PBS and mounted in glycerol-PBS. Duplicate sections were reacted with the fluorescein-conjugated $\mathrm{Ig}$ fraction of rabbit anti-BGG (Cappel N. L. Laboratories, Inc., Cochranville, Pa.), goat anti-rabbit Ig (Calbiochem-Behring Corp., American Hoechst Corp., San Diego, Calif.), and goat anti-mouse C3. (By double immunodiffusion, the fluorescent antisera were found to precipitate specifically and well with all the BGG and rabbit gammaglobulin (RGG) preparations used.) Kidneys 


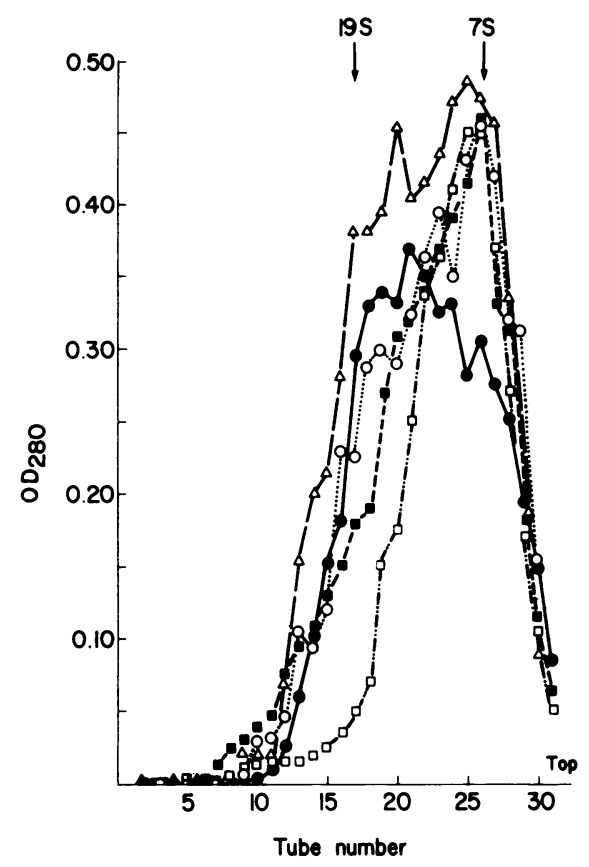

FIgURE 2 Sucrose density gradient ultracentrifugation of five of the immune complexes. To facilitate comparison, the results have been normalized to roughly comparable areas under the immune complex peaks and to $7 \mathrm{~S} \mathrm{IgG} \mathrm{appearing} \mathrm{at}$ tube 26 . The arrows indicate the positions of the $7 \mathrm{~S}$ and $19 \mathrm{~S}$ markers. The large amount of $7 \mathrm{~S}$ sedimenting material is due to some uncomplexed excess BGG antigen and normal (nonanti-BGG) RGG. Symbols for the various IC: $\mathbf{\square}$, group 1; $\square$, group $2 ; \Delta$, group $4 ; 0$, group $9 ; \bigcirc$, group 10

from normal control mice incubated with these reagents showed only trace mesangial staining in a few glomeruli or no fluorescence. Some sections were examined by indirect immunofluorescence with sheep anti-mouse C.3 (generously donated by Dr. Victor Nussenzweig) followed by fuoresceinconjugated rabbit anti-goat Ig (Calbiochem-Behring Corp.), the latter having been shown to cross-react strongly with sheep Ig. The coded slides were examined in a Leitz Dialux fluorescence microscope (E. Leitz Inc., Rockleigh, N. J.). The intensity of staining in the mesangium and glomerular basement membrane was semiquantitatively graded as - , no or trace staining; $1+$, definite but small amounts of staining in some glomeruli; 2+, moderately bright staining in most glomeruli; or $3+$, maximal staining. Extraglomerular staining of tubular basement membranes was also noted. For electron microscopy, $0.5 \mathrm{~mm}^{3}$ blocks were fixed by immersion in $2.5 \%$ glutaraldehyde in $0.2 \mathrm{M}$ cacodylate buffer, $\mathrm{pH} 7.4$, or in Karnovsky's fixative (29), postfixed in $1 \%$ osmium, stained enbloc in uranyl acetate, dehydrated, and embedded in Epon 812 (Shell Chemical Co., Houston, Tex). Thin sections of 2-4 glomeruli from representative groups of mice were double-stained with uranyl acetate and lead citrate and examined and photographed in a Zeiss EM 10A microscope (Carl Zeiss Inc., New York).

\section{RESULTS}

The results are summarized in Table I. By light microscopy, glomeruli appeared normal in all animals.
Controls injected with a mixture of normal BGG and normal RGG in amounts equivalent to the injected IC showed minimal staining for RGG or BGG, only in the mesangium, on immunofluorescence examination (group 11). Those given a mixture of noncomplexed chemically modified cationic Ag and normal RGG (group 12) had BGG but not RGG diffusely bound to glomerular (and tubular) basement membranes indicating that free cationic BGG uncomplexed to $\mathrm{Ab}$ could be bound purely on the basis of positive charge.

Striking differences were observed by immunofluorescence in the amount and distribution of IC in the glomeruli of animals given electrophoretically heterogeneous or anionic IC compared to those given cationic IC. Granular deposits of both BGG and RGG were present in the mesangium but not in the peripheral glomerular basement membranes from mice given heterogeneous (group 1) or anionic (groups 2 and 3) IC (Fig. 3a-d). In contrast, in animals given the most cationic IC (groups 4-6), both BGG and RGG were bound in a diffuse, uniform, smooth pattern in all glomeruli along the peripheral basement membranes (Fig. $3 e$ and $f$ ). The presence of both $\mathrm{Ag}$ and $\mathrm{Ab}$ in the same distribution in the glomerular basement membranes indicates that these were present as IC. This was true only when the IC were prepared with the chemically modified cationic $\mathrm{Ag}$ and focused cationic or neutral fractions of $\mathrm{Ab}$, or with the unfocused heterogeneous $\mathrm{Ab}$ (groups 4-6) but not when IC were prepared with the focused anionic fraction of $\mathrm{Ab}$ (Fig. $3 \mathrm{~g}$ and $\mathrm{h}$; Table I, group 10). In the last case, IC deposition did not occur since only BGG was fixed to the glomerular basement membranes in a manner similar to that seen in controls (group 12) when noncomplexed cationic Ag was injected. The finding of BGG alone in group 10 is explained by the fact that IC were prepared in $\mathrm{Ag}$ excess with some free cationic $\mathrm{Ag}$ available for binding to the glomerular basement membrane. IC prepared with the focused cationic fractions of $\mathrm{Ag}$ and $\mathrm{Ab}$ (group 7), with the heterogeneous unfocused $\mathrm{Ag}$ and the focused cationic fraction of $\mathrm{Ab}$ (group 8), or with the chemically modified anionic Ag and the focused cationic fraction of $\mathrm{Ab}$ (group 9) did not localize in the glomerular basement membane since neither BGG nor RGG was detectable there by immunofluorescence. Mouse third component of complement was not associated with the IC, which can perhaps be attributed to the fact that the IC used were prepared in Ag excess; such complexes are known to fix complement poorly.

It should be emphasized that the IC were prepared in $\mathrm{Ag}$ excess. In the limiting case for a bivalent $\operatorname{IgG~Ab}$ there would be two molecules of $\mathrm{Ag}$ per molecule of $\mathrm{Ab}$ in the IC. The charge of the Ag is, therefore, the major determinant of the net charge of the IC. The failure of the IC of groups 7-9 to localize in the glomerular base- 

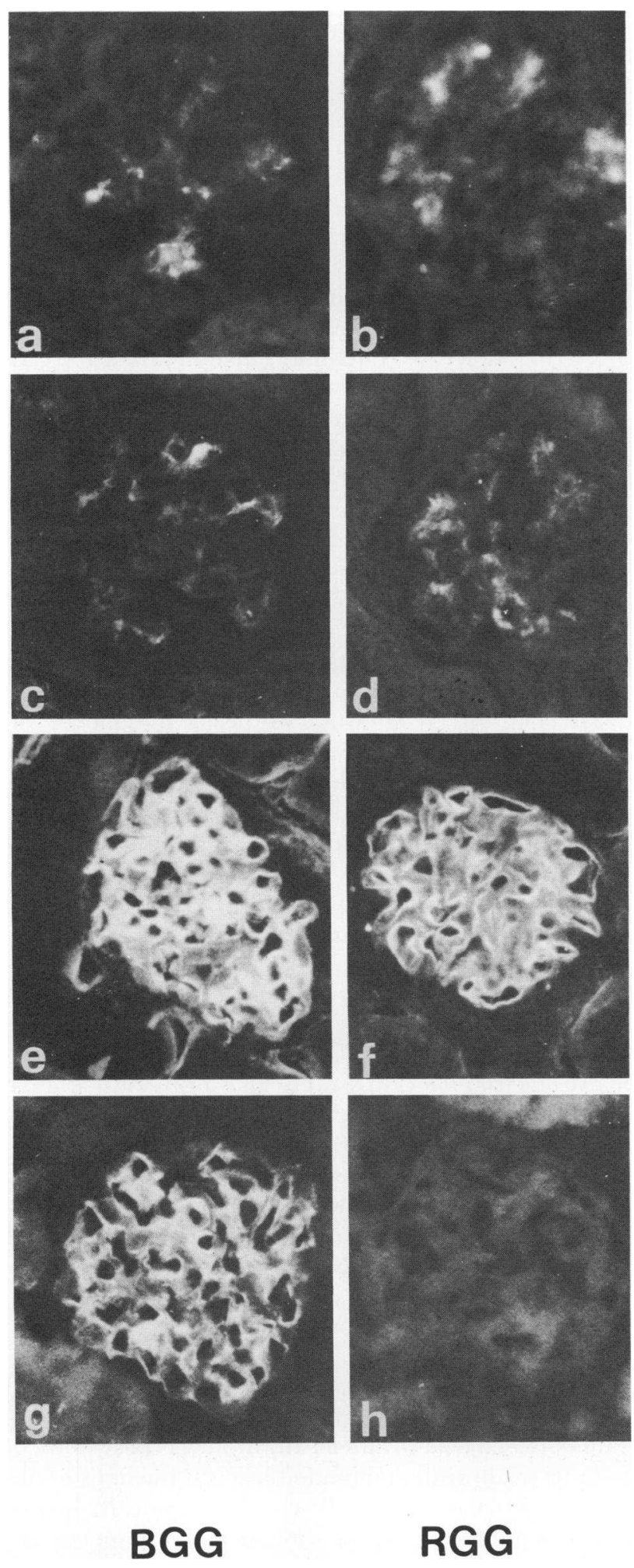

\section{RGG}

FIGURE 3 Immunofluorescence with fluorescein-labeled antiBGG (left) and anti-RGG (right). Glomeruli from mice receiving IC prepared from heterogeneous $\mathrm{Ag}$ and $\mathrm{Ab}$, group 1, ( $\mathrm{a}$ and $\mathrm{b}$ ) or anionic $\mathrm{Ag}$ and $\mathrm{Ab}$, group 2, (c and d) demon- ment membrane can thus be explained by their being, in aggregate, less cationic than those of groups 4-6, groups 7-9 not having been made with the most cationic Ag preparation, the chemically modified one.

BGG but not RGG localized in the tubular basement membranes only in mice given chemically modified cationic Ag either as ICs or as noncomplexed protein indicating that IC deposition did not occur at this site and that BGG was deposited there on the basis of positive charge alone. The staining was diffuse and generally smooth, but a fine granular pattern was discernible in tangential views (Fig. 4).

Ultrastructurally, there were no deposits in the glomerular basement membranes of mice given electrophoretically heterogeneous (group 1) or anionic (group 2) IC (Fig. 5a and b). In those given IC formed with the chemically modified cationic Ag (groups 4-6 and 10), deposits were present in subendothelial and subepithelial sites. In the latter location, deposits never abutted on the overlying cell membrane. The deposits were most numerous and largest in mice given IC formed with the focused cationic fraction of $\mathrm{Ab}$ (Fig. $5 \mathrm{c}$; group 4) as compared to IC formed with neutral (Fig. 5d; group 5) or heterogeneous fractions of $\mathrm{Ab}$, suggesting that the more highly positively charged IC accumulate in greater amounts in the glomerular basement membrane. This differential accumulation was most pronounced $(3+$ vs. $1+)$ in the subepithelial location. The noncomplexed cationic Ag was also seen as electron-dense deposits in similar locations, i.e., in subendothelial and subepithelial sites (Fig. 5e; group 12). These deposits ultrastructurally resembled those formed by IC, which could only be distinguished by the immunofluorescence findings demonstrating RGG. In mice given IC prepared with chemically modified cationic Ag and the focused anionic fraction of $\mathrm{Ab}$ (group 10), glomerular basement membrane deposits, though present, were smaller and less regular than in those given IC prepared with cationic or neutral $\mathrm{Ab}$ (groups 4 and 5). As determined by the absence of RGG on immunofluorescence, group 10 basement membrane deposits were not IC but instead were excess cationic Ag unbound to Ab. The deposits were less numerous than in control mice given un-

strate respectively $2+$ and $1+$ mesangial deposits of BGG and RGG. Glomeruli from mice injected with cationic IC prepared from chemically modified cationic Ag and the focused cationic Ab, group 4, (e and $f$ ) show diffuse $3+$ binding of BGG and RGG in peripheral glomerular basement membrane. The deposits appear linear, but at the ultrastructural level are discrete and granular (Fig. 5c). In mice given IC prepared with chemically modified cationic $\mathrm{Ag}$ and focused anionic $\mathrm{Ab}$, group 10, (g and $\mathrm{h}$ ), there is no RGG in glomeruli $(\mathrm{h})$ indicating that IC deposition has not occurred. The localization of BGG $(3+)$ alone $(\mathrm{g})$ is due to deposition of excess uncomplexed cationic Ag. $\times 400$. 


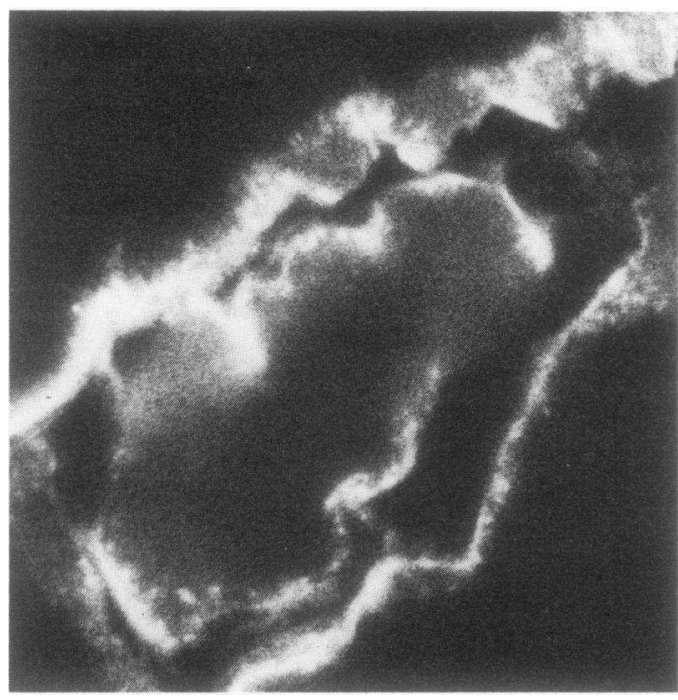

FIGURE 4 Kidney from a mouse receiving chemically modified cationic BGG and normal RGG. Finely granular deposits of BGG are present in tubular basement membranes. There was no staining for RGG (not shown). $\times 2,960$.

complexed cationic Ag (group 12) presumably because there was less uncomplexed Ag available for binding to anionic sites in the glomerular basement membrane. Electron dense deposits in the tubular basement membranes (Fig. 5f; group 12) were due to binding of cationic Ag only as determined by the absence of RGG on immunofluorescence.

\section{DISCUSSION}

Our results provide the first evidence that passively administered IC can lead to diffuse and prominent deposition in the glomerular basement membrane on the basis of IC charge. This was demonstrated by the fact that cationic, but not anionic or native heterogeneous IC injected intravenously into mice, were heavily deposited in subendothelial and subepithelial layers. ${ }^{2}$ Differences in the size of the ICs are unlikely to have played a significant role since all preparations of IC sedimented in the same broad range of 9-25S and there were no distinct peaks whose presence or absence correlated with the ability to deposit in glomeruli. Previous attempts in the passive serum sickness model have generally failed to demonstrate convincingly that intravenously injected IC can deposit prominently in subepithelial sites (14-20). Factors such as amount $(14,18,19)$, size $(20)$ and affinity $(18)$, or modification of IC by reduction and alkylation (17) have generally had no effect on subepithelial glomeru-

\footnotetext{
${ }^{2}$ In this paper, subepithelial layer or space is synonymous with the lamina rara externa of the glomerular basement membrane.
}

lar basement membrane localization. In mice given low avidity complexes, subepithelial deposits have been described (21) but in these experiments much larger and more numerous doses of IC were used and deposition appeared less extensive than in the present work.

Several lines of evidence suggest that intrinsic properties of the glomerulus could favor the trapping of IC in the glomerular basement membrane. Anionic sites in the subendothelial and subepithelial layers demonstrated by cationic probes $(22,23)$ and characterized enzymatically and biochemically as sulfated glycosaminoglycans containing heparan sulfate $(30,31)$ provide a structural basis for the binding of positively charged molecules. Electrostatic interactions are known to occur under physiologic conditions between negatively charged sulfated polysaccharides and positively charged proteins (32). The anionic sites in the glomerular basement membrane have been shown to bind lysozyme (22), cationic ferritin $(23,25)$, protamine (33), and protamine-heparin complexes formed by perfusion with protamine followed by heparin (34). These considerations suggested that charge interactions between the anionic sites and IC could be a basis for deposition.

To test this hypothesis, we used the model of passive serum sickness. Rabbits were used as a source of $\mathrm{Ab}$ because their response after hyperimmunization to foreign protein is largely IgG, and rabbit IgG appears not to contain subclasses. Thus, a differential distribution of subclasses in the various focused fractions should not complicate interpretations. BGG was chosen as the $\mathrm{Ag}$ for two reasons. It is readily available and has only two subclasses (35); IgGl and IgG2 were both present in all fractions used.

Two methods of varying net charge of IC were employed. Isoelectric focusing of Igs, which are normally electrophoretically quite heterogeneous, affords a means of fractionation that does not involve or require modification of the native structure. However, it was not possible with focused Ag and focused Ab to prepare IC that were sufficiently cationic to deposit in the glomerular basement membrane. Consequently, chemical modifications of BGG were used to provide more highly cationic (and also anionic) Ag. The methods used each provide a net alteration of two charges for each side chain modified. Corresponding treatment of the anti-BGG $\mathrm{Ab}$ greatly inhibited its ability to precipitate with BGG Ag. Hence, the relationship between charge and localization was best studied with IC made with unmodified focused fractions of $\mathrm{Ab}$ (pI, 2.5-5.0; 6.5-7.0; > 8.0) and the chemically modified cationic or anionic Ag. Pronounced differences according to IC charge were observed: the most cationic IC clearly localized in the peripheral glomerular basement membrane while anionic and heterogeneous IC did not.

The ultrastructural distribution of the cationic IC was 

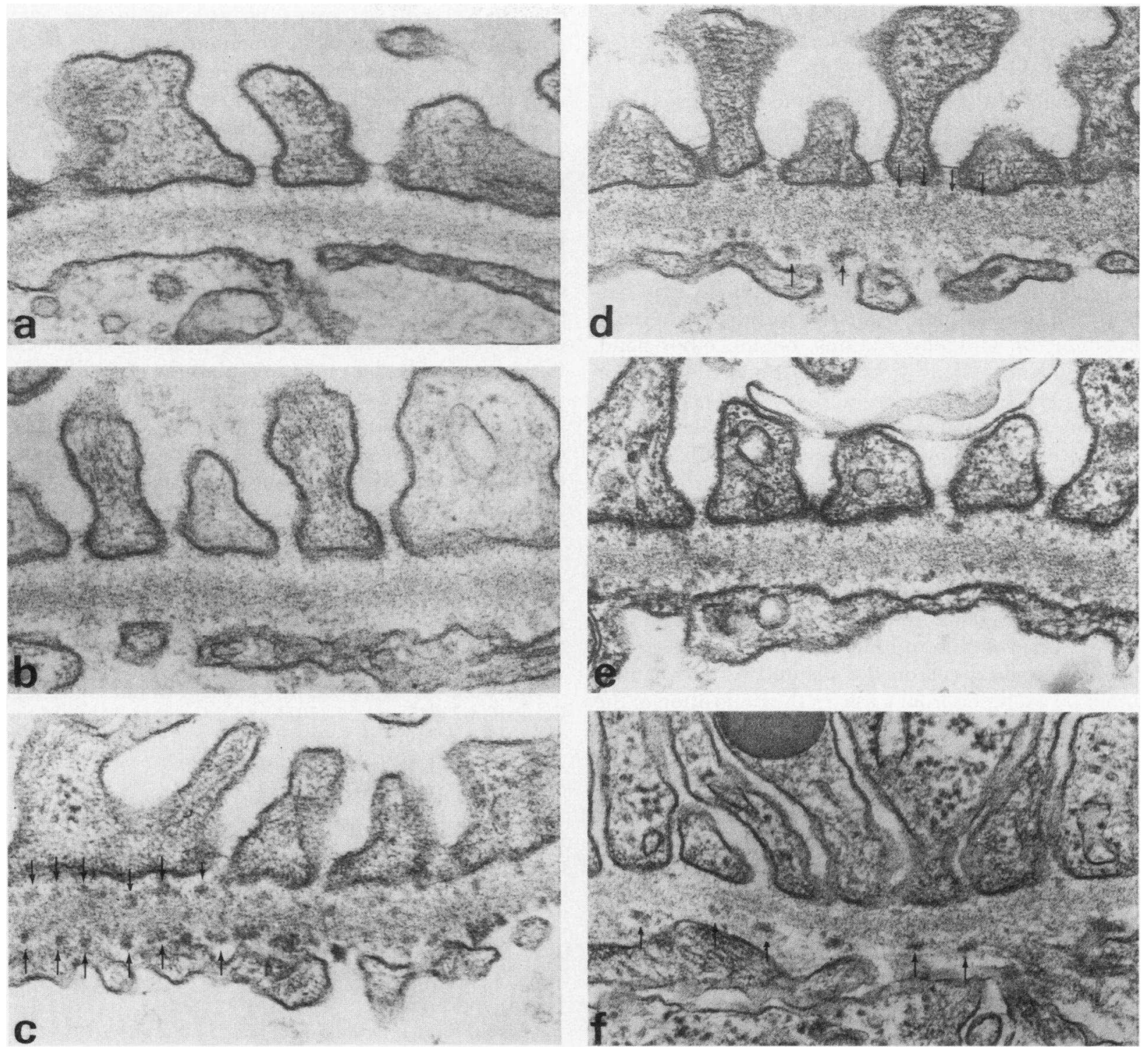

FIGURE 5 Electron micrographs of peripheral glomerular basement membrane of mice given native heterogeneous IC, group 1, (a) and anionic IC prepared from the chemically modified anionic BGG and the focused anionic Ab to BGG, group 2, (b) show no deposits in subendothelial or subepithelial sites. (c) Glomerular basement membrane from a mouse given cationic IC made from chemically modified cationic BGG and focused cationic Ab (group 4) demonstrates deposits in subepithelial and subendothelial sites (arrows). Similar, though smaller deposits (arrows) are present in glomerular basement membrane from an animal injected with IC prepared from chemically modified cationic BGG and focused neutral Ab, group 5, (d); deposits by immunofluorescence were shown to contain both BGG and RGG. Control mouse (e) injected with chemically modified cationic BGG and normal RGG (group 12) also shows deposits, which contained BGG but not RGG by immunofluorescence. Wall of proximal tubule (f) shows deposits (arrows) in tubular basement membrane in a mouse given chemically modified cationic BGG and normal RGG (group 12). ×61,000.

similar to that observed after the administration of cationic BGG alone, or cationic ferritin (23), or in vitro perfusion of kidneys with lysozyme and ruthenium red $(22,23)$, namely in the layers of the glomerular basement membrane where anionic sites occur. All of these cationic substances are known to bind to anionic sites in the inner and outer layers of the glomerular basement membrane. These ultrastructural similarities strongly suggest that cationic IC bind to anionic sites in the same manner. In the present study, the most 
cationic IC (group 4) localized even more extensively in the subepithelial zones than did free cationic BGG (group 12), 3+ vs. 1+.

Although the significance of charge of the circulating immune reactants in the present experiments seems clear, the sequence by which the deposition occurs is not certain. In the past, the general failure to find deposits in subepithelial spaces after the intravenous injection of IC, though such deposits are readily found after passive administration of $\mathrm{Ab}$ to renal tubular $\mathrm{Ag}$ in the model of passive Heymann nephritis, has suggested that subepithelial deposits result from in situ formation (9). Our observations, on the other hand, support the idea that direct deposition of circulating IC may be a mechanism in some systems. In our experiments $\mathrm{Ag}$ and $\mathrm{Ab}$ were both deposited in the same sites of the glomerular basement membrane soon after injection of the most cationic IC. Furthermore, when mice were sacrificed immediately after injection of cationic IC, both BGG and RGG were present. Thus, at no time was cationic Ag detectable in the absence of $\mathrm{Ab}$ after injection of cationic IC.

However, since the free chemically modified cationic $\mathrm{Ag}$ by itself was able to bind to glomerular basement membrane, variations on the planted Ag mechanism cannot be excluded from a role in the deposition of the cationic IC. ICs containing occasional free Ab combining sites could bind to excess free cationic Ag previously deposited alone. If this were so, IC formed from the chemically modified cationic $\mathrm{Ag}$ and the anionic focused $\mathrm{Ab}$ might have lodged equally well as those formed from the cationic or neutral fractions of focused $\mathrm{Ab}$. This was not the case. Alternatively, free $\mathrm{Ab}$ dissociated from circulating IC could cross the glomerular basement membrane and bind to the planted cationic Ag. In this event, free cationic Ab would be more likely to cross the basement membrane than free anionic $\mathrm{Ab}$. This possibility is also considered less likely because the $\mathrm{Ab}$ are in $\mathrm{Ag}$ excess and are bivalent, which favors multipoint binding. Thus, it is improbable for a whole $\mathrm{Ab}$ molecule to diffuse completely away from the IC. It must be emphasized that the various mechanisms just discussed for leading to the deposition of the cationic IC are by no means mutually exclusive.

Under other circumstances, IC containing cationic Ag could form in situ in the glomerular basement membrane. Any sufficiently cationic substance, whether of endogenous or exogenous origin, that reaches the circulation could potentially bind through electrostatic interaction, independent of any immune mechanism. If the process were short lived, it would not necessarily be deleterious. However, if the cationic deposit were antigenic, an $\mathrm{Ab}$ response could ensue and the resulting IC formed in situ could bring about an immunologically mediated inflammatory response.

The binding of cationic BGG alone demonstrates that Ig as well as other cationic molecules may accumulate in glomeruli on a nonimmunological basis. Such a mechanism may conceivably account for the accumulation of monoclonal Igs in glomeruli and tubular basement membranes in some patients with monoclonal gammopathies in whom evidence for an immune mechanism is lacking $(36,37)$.

Our studies emphasize that anionic sites in the glomerular basement membranes can interact electrostatically with IC or free Ag under physiologic conditions. Other nonimmunologic mechanisms for binding potential $\mathrm{Ag}$ are illustrated by the interaction of the lectin concanavalin A with specific glycoprotein in the glomerular basement membrane (11) and by the interaction of DNA, a polyanion, with collagen of isolated glomerular basement membrane (38). Thus, it is quite likely that a multiplicity of factors can contribute to IC localization in the glomerulus. These remain to be demonstrated in human forms of glomerulonephritis.

\section{ACKNOWLEDGMENTS}

We thank Ms. Virginia Meyer, Ms. Susan Buda, and Ms. Beatrice Delgado for excellent technical assistance, and Ms. Neva Harper, Ms. Gwynne Nemcek, and Ms. Alice Brzytwa who typed the manuscript.

This work was supported by National Institutes of Health Grants CA-23885 and AI-15071 and a Grant-in-Aid from the Kidney Foundation of New York, Inc.

\section{REFERENCES}

1. Germuth, F. G. 1953. A comparative histologic and immunologic study in rabbits of induced hypersensitivity of the serum sickness type. J. Exp. Med. 97: 257-282.

2. Germuth, F. G., C. Flanagan, and M. R. Montenegro. 1957. The relationships between the chemical nature of the antigen, antigen dosage, rate of antibody synthesis and the occurrence of arteritis and glomerulonephritis in experimental hypersensitivity. Bull. Johns Hopkins Hosp. 101: $149-169$.

3. Dixon, F. J., J. D. Feldman, and J. J. Vasquez. 1961. Experimental glomerulonephritis. The pathogenesis of a laboratory model resembling the spectrum of human glomerulonephritis. J. Exp. Med. 113: 899-920.

4. Germuth, F. G., L. B. Senterfit, and G. R. Dreesman. 1972. Immune complex disease. $V$. The nature of the circulating complexes associated with glomerular alterations in the chronic BSA-rabbit system. Hopkins Med.J. 130: 344-357.

5. Fleuren, G. J., R. V. D. Lee, H. A. Greben, B. J. C. Van Damme, and Ph. J. Hoedemaeker. 1978. Experimental glomerulonephritis in the rat induced by antibodies directed against tubular antigens IV. Investigations into the pathogenesis of the model. Lab. Invest. 38: 496-501.

6. Van Damme, B. J. C., G. J. Fleuren, W. W. Bakker, R. L. Vernier, and $\mathrm{Ph}$. J. Hoedemaeker. 1978. Experimental glomerulonephritis in the rat induced by antibodies directed against tubular antigens V. Fixed glomerular antigens in the pathogenesis of heterologous immune complex glomerulonephritis. Lab. Invest. 38: 502-510.

7. Couser, W. G., D. R. Steinmuller, N. M. Stilmant, D. J. Salant, and L. M. Lowenstein. 1978. Experimental glomerulonephritis in the isolated perfused rat kidney. $J$. Clin. Invest. 62: 1275-1287. 
8. Salant, D. J., S. Belok, M. M. Stilmant, C. Darby, and W. G. Couser. 1979. Determinants of glomerular localization of subepithelial immune deposits: effects of altered antigen to antibody ratio, steroids, vasoactive amine antagonists, and aminonucleoside of puromycin on passive Heymann nephritis in rats. Lab. Invest. 41: 89-99.

9. Couser, W. G., and D. J. Salant. 1980. In situ immume complex formation and glomerular injury. Kidney Int. 17: $1-13$.

10. van Es, L. A., A. P. R. Blok, L. Schoenfeld, and R. J. Glassock. 1977. Chronic nephritis induced by antibodies reacting with glomerularbound immune complexes. Kidney Int. 11: 106-115.

11. Golbus, S., and C. B. Wilson. 1979. Experimental glomerulonephritis induced by in situ formation of immune complexes in glomerular capillary wall. Kidney Int. 16: $148-157$.

12. McCluskey, R. T., B. Benacerraf, and F. Miller. 1962. Passive acute glomerulonephritis induced by antigenantibody complexes solubilized in hapten excess. Proc. Soc. Exp. Biol. Med. 111: 764-768.

13. Mellors, R. C., and W. J. Brzosko. 1962. Studies in molecular pathology: I. Localization and pathogenic role of heterologous immune complexes. J. Exp. Med. 115: 891-903.

14. Okumura, K., Y. Kondo, and T. Tada. 1971. Studies on passive serum sickness I. The glomerular fine structure of serum sickness nephritis induced by preformed antigen-antibody complexes in the mouse. Lab. Invest. 24: 383-391.

15. Wright, N. G., W. I. Morrison, H. Thompson, and H. J. C. Cornwell. 1973. Experimental adenovirus immune complex glomerulonephritis. Br.J. Exp. Pathol. 54: 628-633.

16. Ford, P. M. 1975. The effect of manipulation of reticuloendothelial system activity on glomerular deposition of aggregated protein and immune complexes in two different strains of mice. Br. J. Exp. Pathol. 56: 523-529.

17. Haakenstad, A. O., G. E. Striker, and M. Mannik, 1976. The glomerular deposition of soluble immune complexes prepared with reduced and aklylated antibodies and with intact antibodies in mice. Lab. Invest. 35: 293-301.

18. Koyama, A., Y. Niwa, H. Shigematsu, M. Taniguchi, and T. Tada. 1978. Studies on passive serum sickness II. Factors determining the localization of antigen-antibody complexes in the murine renal glomerulus. Lab. Invest. 38: 253-262.

19. Germuth, F. G., E. Rodriguez, C. A. Lorelle, E. I. Trump, L. Milano, and O. Wise. 1979. Passive immune complex glomerulonephritis in mice: models for various lesions found in human disease I. High avidity complexes and mesangiopathic glomerulonephritis. Lab. Invest. 41: 360-365.

20. Kijlstra, A., M. R. Daha, and L. A. van Es. 1979. Immune complexes in experimental glomerulonephritis. Behring Inst. Mitt. No. 64: 45-53.

21. Germuth, F. G., E. Rodriguez, C. A. Lorelle, E. Trump, L. L. Milano, and O. Wise. 1979. Passive immune complex glomerulonephritis in mice: models for various lesions found in human disease II. Low avidity complexes and diffuse proliferative glomerulonephritis with subepithelial deposits. Lab. Invest. 41: 366-371.

22. Caulfield, J. P., and M. G. Farquhar. 1976. Distribution of anionic sites in glomerular basement membranes: their possible role in filtration. Proc. Natl. Acad. Sci. U. S. A. 73: 1646-1650.
23. Kanwar, Y. S., and M. G. Farquhar. 1976. Anionic sites in the glomerular basement membrane. In vivo and in vitro localization to the laminae rarae by cationic probes. $J$. Cell Biol. 81: 137-153.

24. Bremner, B. M., M. P. Bohrer, C. Baylis, and W. M. Deen 1977. Determinants of glomerular permselectivity: insights derived from observations in vivo. Kidney Int. 12 229-237.

25. Rennke, H. G., R. S. Cotran, and M. V. Venkatachalam. 1975. Role of molecular charge in glomerular permeability. Tracer studies with cationic ferritins. J. Cell Biol. 67: 638-646.

26. Butler, P. J. G., J. I. Harris, B. S. Hartley, and R. Leberman. 1969. The use of maleic anhydride for the reversible blocking of amino groups in polypeptide chains. Biochem. J. 112: 679-689.

27. Dannon, D., L. Goldstein, Y. Marikovsky, and E. Skultelsky. 1972. Use of cationized ferritin as a label of negative charges on cell surfaces. J. Ultrastruct. Res. 38 $500-510$

28. Heidelberger, M., F. E. Kendall, and C. M. Soo Hoo. 1933. Quantitative studies on the precipitin reaction. Antibody production in rabbits injected with an azo protein. $J$. Exp. Med. 58: 137-152.

29. Karnovsky, M. J. 1965. A formaldehyde-glutaraldehyde fixative of high osmolality for use in electron microscopy. J. Cell Biol. 27: 137a. (Abstr.)

30. Kanwar, Y. S., and M. G. Farquhar. 1979. Presence of heparan sulfate in glomerular basement membrane. Proc. Natl. Acad. Sci. U. S. A. 76: 1303-1307.

31. Kanwar, Y. S., and M. G. Farquhar. 1979. Isolation of glycosaminoglycans (heparan sulfate) from glomerular basement membranes. Proc. Natl. Acad. Sci. U. S. A. 76: 4493-4497.

32. Comper, W. D., and T. C. Laurent. 1978. Physiological function of connective tissue polysaccharides. Physiol. Rev. 58: 255-315.

33. Seiler, M. W., N. G. Rennke, M. A. Venkatachalam, and R. S. Cotran. 1977. Pathogenesis of polycation-induced alterations ("fusion") of glomerular epithelium. Lab. Invest. 36: 48-61.

34. Sharon, Z., M. M. Schwartz, and E. J. Lewis. 1977. The glomerular localization and transport of aggregated protamine-heparin complexes. Lab. Invest. 37: 43-52.

35. Aaland, O., D. Blakeslee, J. E. Butler, J. R. Duncan, M. J. Freeman, R. Jenness, J. M. Kehoe, J-P. Mach, J. Rapacz, J-P. Vaerman, and A. J. Winter. 1971. Proposed nomenclature for the immunoglobulins of the domesticated bovidae cattle (bos taurus) sheep (ovis aries) goats (capra hircus). Canadian J. Comp. Med. 35: 346-348.

36. Beaufils, M., and L. Morel-Maroger. 1978. Pathogenesis of renal disease in monoclonal gammopathies: Current concepts. Nephron. 20: 125-131.

37. Gallo, G. R., H. D. Feiner, L. A. Katz, G. M. Feldman, E. B. Correa, J. V. Chuba, and J. N. Buxbaum. 1980. Nodular glomerulopathy associated with nonamyloidotic kappa light chain deposits and excess immunoglobulin light chain synthesis. Am. J. Pathol. 99: 621-644.

38. Izui, S., P. H. Lambert, and P. A. Miescher. 1976. In vitro demonstration of a particular affinity of glomerular basement membrane and collagen for DNA. A possible basis for a local formation of DNA-anti-DNA complexes in systemic lupus-erythematosus.J. Exp. Med. 144: 428-443. 